\title{
Ajuste dos modelos Gompertz e Logístico aos dados de crescimento de frutos de coqueiro anão verde
}

\author{
The fit Gompertz and Logístic models to the growth data of green dwarf coconut fruits
}

\author{
Thalita Kelen Leal do Prado ${ }^{I^{*}}$ Taciana Villela Savian $^{\text {II }}$ Joel Augusto Muniz ${ }^{\mathrm{I}}$
}

RESUMO

O objetivo do trabalho foi estudar o ajuste dos modelos não-lineares, gompertz e logístico, na descrição do desenvolvimento de frutos de coqueiro da variedade anão verde, com base nos dados de diâmetros externos, longitudinal e transversal. Os resultados indicaram que tanto para o diâmetro longitudinal externo (DEL) como para o diâmetro transversal externo (DET), o modelo logístico foi o mais adequado na descrição do crescimento do fruto. $O$ ajuste dos modelos, para ambas as variáveis, apresentou autocorrelação residual positiva. Para o DEL o resíduo foi modelado segundo um processo autoregressivo de primeira ordem (ARI) e para a variável DET o processo autoregressivo considerado foi de segunda ordem (AR2). A consideração do processo autoregressivo (AR1 ou $A R 2)$ resultou em estimativas dos parâmetros mais precisas. De acordo com o modelo logístico, o fruto do coqueiro anão verde, totalmente desenvolvido, tem diâmetros externos longitudinal e transversal, respectivamente, de $21,56 \mathrm{~cm}$ e $15,35 \mathrm{~cm}$.

Palavras-chave: modelo logístico, modelo gompertz, erros correlacionados.

\section{ABSTRACT}

The aim of this study was to evaluate the quality fit of nonlinear models, gompertz and logistic data outside diameter, longitudinal (DEL) and transverse (DET) fruits of green dwarf coconut trees, as well as verification of the existence of residual autocorrelation. The results showed that for both, DEL and DET, the logistic model is most appropriate for describing the fruits growth. The fit of the models, for both variables, showed positive residual autocorrelation. For DEL, the residual was modeled by a first order autoregressive process, and for DET, the autoregressive process was considered of second order. Consideration of the autoregressive process resulted in more accurate estimates of the parameters. According to the logistic model, the fruit of the dwarf green, fully developed, have external diameters, longitudinal and transverse, respectively, of $21.56 \mathrm{~cm}$ and $15.35 \mathrm{~cm}$.

Key words: logistic model, gompertz model, correlated errors.

\section{INTRODUÇÃO}

Ocoqueiro(Cocos nuficera L.) davariedade anão caracteriza-se por ser uma planta de porte baixo, precoce, com reprodução predominantemente por autofecundação, monóica, produzindo flores unissexuais em uma mesma inflorescência do tipo paniculada e axilar (MEDINA, 1980). Esta variedade originou-se, provavelmente, de uma mutação ocorrida na variedade Gigante e apresenta frutos com pouca polpa e água muito saborosa destinada ao consumo, seja sob a forma in natura ou processadas em agroindústrias (MACIEL, 2009).

De acordo com BITENCOURT \& PEDROTI (2008), o coqueiro anão verde constituise na variedade de coqueiro mais utilizada comercialmente no Brasil, para produção de água de coco, com qualidade sensorial superior às das demais cultivares. Os frutos destinados ao consumo in natura de sua água devem ser colhidos, principalmente, entre o sexto e o sétimo mês, após a abertura natural da inflorescência, independente da cultivar considerada. Nessa idade ocorrem os maiores valores para peso de fruto, produção de água, teores de frutose, glicose, grau brix e sais minerais, principalmente potássio, os

'Departamento de Ciências Exatas, Universidade Federal de Lavras (UFLA), 37200-000, Lavras, MG, Brasil. E-mail: tkprado1@ yahoo.com.br.

*Autor para correspondência.

"Departamento de Ciências Exatas, Universidade de São Paulo (USP), Piracicaba, SP, Brasil. 
quais conferem melhor sabor à água de coco. Os frutos colhidos precocemente apresentam água menos doce, e naqueles colhidos tardiamente ocorrem aumentos nos teores de gordura, ocasionando um sabor rançoso a água de coco.

ARAGÃO (2007) relata que o ponto ideal de colheita do fruto está associado a uma série de indicadores morfológicos relacionados à idade, ao tamanho do fruto e a presença de determinadas substâncias na água. No decorrer do desenvolvimento, o fruto de coco inicialmente cresce mais em comprimento do que em largura, ou seja, com o diâmetro transversal menor que o diâmetro longitudinal, atingindo o tamanho e peso máximos aos seis meses de idade.

Os modelos estatísticos que melhor descrevem as curvas de crescimento, de um modo geral, são os modelos de regressão não-linear e de forma semelhante aos modelos lineares, o processo de estimação dos parâmetros pode ser obtido pela minimização da soma de quadrados dos erros, obtendo-se o sistema de equações normais não-linear, o qual não apresenta uma solução explícita e é obtido por processos iterativos (SOUZA, 1998).

Os métodos iterativos mais conhecidos são: o método Steepest-Descent ou método do Gradiente, o método de Marquardt e o método de Gauss-Newton, sendo este último um dos mais utilizado pelos autores em estudos de modelos nãolineares (SAVIAN \& MUNIZ, 2007; PEREIRA et al., 2005; MAZZINI et al. 2005).

Geralmente, em modelos de regressão, assume-se que os erros não estão correlacionados, o que não é apropriado quando se trabalha com séries cronológicas de dados (MORETTIN \& TOLOI, 2004). A não consideração de pressuposições associadas ao modelo pode ocasionar a obtenção de estimativas viesadas e a subestimação das variâncias dos parâmetros. Segundo MOKKADEM (1985), quando os resíduos são autocorrelacionados, as estimativas de mínimos quadrados ordinários dos parâmetros não são eficientes, isto é, não apresentam variância mínima, além de seu erro-padrão ser viesado, o que conduz a testes e intervalos de confiança incorretos. Se a autocorrelação for positiva, os erros padrões serão substimados e, consequentemente, os valores da estatística t, superestimados. Se a autocorrelação for negativa, os erros padrões serão superestimados e o valor da estatística t, subestimado. Portanto a autocorrelação positiva é mais danosa, porque existirá, no caso do teste " $t$ ", o risco de rejeitar-se a hipótese nula de ausência do efeito, quando deveria aceita-la.
PEREIRA et al. (2005), em estudo sobre a predição do nitrogênio mineralizado utilizando modelos não-lineares, incorporaram a autocorrelação residual e encontraram estimativas mais precisas para os parâmetros envolvidos nos modelos. Na avaliação do crescimento de fêmeas Hereford com o modelos logístico difásico, MENDES et al. (2008) consideraram estrutura autoregressiva de primeira e segunda ordem para o vetor residual e obtiveram ajustes mais eficientes do que no ajuste dos modelos sem autocorrelação.

Este trabalho objetivou avaliar as propriedades dos estimadores dos parâmetros dos modelos não lineares, gompertz e logístico, considerando no ajuste a estrutura de erros independentes e autocorrelacionados. Após a escolha da estrutura residual mais adequada, objetivouse comparar o ajuste dos modelos na descrição do crescimento de frutos de coqueiro anão verde em diferentes idades.

\section{MATERIAL E MÉTODOS}

Os dados utilizados para o ajuste dos modelos foram obtidos de um experimento (BENASSI, 2006) em que foi mensurado, dentre outras características, os diâmetros externos, longitudinal (DEL) e transversal (DET), de frutos de coqueiro da variedade anão verde. O trabalho de campo foi realizado em um coqueiral (Cocos nucifera L.) variedade anão verde, com idade de sete anos, instalado na Estação Experimental de Citricultura de Bebedouro/SP - EECB. O espaçamento utilizado foi de 7,0m entre linhas e $6,0 \mathrm{~m}$ entre plantas, em disposição triangular. Antes do plantio foi realizada a calagem em área total, objetivando-se elevar a saturação de bases para $65 \%$. As covas foram abertas mecanicamente nas dimensões de 60x60x60cm utilizando-se para seu preparo, 20 litros de esterco de curral curtido, $800 \mathrm{~g}$ de superfosfato simples e $300 \mathrm{~g}$ de calcário dolomítico.

As avaliações foram feitas em oito plantas diferentes, marcando-se uma inflorescência em cada planta, com base nas medidas de três frutos identificados nas regiões apical, mediana e basal do cacho, objetivando maior representatividade amostral, totalizando-se 24 frutos. A primeira medição foi feita no dia da abertura da inflorescência que se deu em 15/04/2004, e as demais, a cada 15 dias nos frutos marcados, encerrando-se em 01/05/2005, totalizando 26 medições. Em cada data foram anotados os diâmetros externos, longitudinal e transversal dos 24 frutos, obtendo-se as médias para o ajuste dos modelos. 
Os modelos não-lineares, logístico (1) e gompertz (2), foram utilizados para estimar os parâmetros da curva de crescimento dos frutos, dados por:

$W_{i}=\frac{A}{1+B\left[\exp \left(-K t_{i}\right)\right]}+u_{i}$

$W_{i}=\operatorname{Aexp}\left\{-B\left[\exp \left(-K t_{i}\right)\right]\right\}+u_{i}$

em que: $u_{i}=\rho_{1} u_{i-1}+\cdots+\rho_{p} u_{i-p}+\varepsilon_{i}$, com $\mathrm{i}=1, \ldots, \mathrm{n}$ e n o número de medições no fruto; $u_{i}$ é o resíduo do ajuste na i-ésima medição; $\rho_{1}$ é o parâmetro autorregressivo de ordem 1; $u_{i-1}$ é o resíduo do ajuste no tempo imediatamente anterior a i-ésima medição; $\rho_{p}$ é o parâmetro autorregressivo de ordem $\mathrm{p} ; u_{i-p}$ é o resíduo do ajuste em $\mathrm{p}$ tempos anterior a i-ésima medição; $\varepsilon_{i}$ é o ruído branco com distribuição normal $N\left(0, \sigma^{2}\right)$, para a estrutura de erros independentes $u_{i}=\varepsilon_{i} ; \mathrm{W}_{i}$ expressa o diâmetro (cm) do fruto na i-esima medição; A é o parâmetro assintótico que corresponde ao diâmetro do fruto à maturação; B corresponde ao parâmetro de locação, sem interpretação biológica; $\mathrm{K}$ determina a taxa de expansão do crescimento $\left(\mathrm{cm} \mathrm{dia}^{-1}\right)$ do fruto, $t_{i}$ refere-se ao tempo na i-ésima medição, dado em dias após a abertura da inflorescência.

No ajuste dos modelos utilizou-se o PROC MODEL do software Statistical Analysis System (SAS, 1999). A verificação da presença de autocorrelação residual foi feita da seguinte forma: primeiramente, realizou-se o ajuste do modelo considerando a estrutura de erros independentes. Para esse vetor de resíduos foi verificada a significância da estatística do teste de Durbin-Watson (DW), que testa a hipótese nula de que os resíduos são não correlacionados contra a hipótese alternativa de que os resíduos seguem um processo autorregressivo de primeira ordem (AR1).

Sendo a estatística do teste significativa, considerando um nível de significância de 5\%, o vetor de resíduos foi utilizado no banco de dados como uma covariável, defasada em uma observação e indexada pelo parâmetro $\rho_{1}$, e os parâmetros do modelo foram estimados conjuntamente. Ao realizar o teste de Durbin-Watson considerando $\rho_{1}$ no modelo, sendo a estatística do teste significativa, o novo vetor de resíduos é utilizado como uma covariável, defasada em duas observações e assim o parâmetro $\rho_{2}$ é incluído no modelo. Devido às características da variável independente, medidas quinzenais do fruto, apenas foram consideradas no estudo as autocorrelações residuais de primeira e segunda ordem, pois a estatística de Durbin-Watson (DW) foi não significativa em cada caso, para ordens superiores de autocorrelação.
A seleção do modelo mais adequado teve como base, além da interpretação biológica dos parâmetros, a qualidade dos ajustes obedecendo aos seguintes critérios:

1. Coeficiente de determinação ajustado $\left(R_{a j}^{2}\right)$, dado por: $R_{a j}^{2}=1-\left[\frac{(n-1)\left(1-R^{2}\right)}{(n-q)}\right]$, em que: $\mathrm{R}^{2}$ é o coeficiente de determinação não ajustado, $\mathrm{n}$ é o número de medições no fruto e q é o número de parâmetros do modelo;

2. Desvio padrão residual (DPR), dado por: $D P R=\sqrt{Q M E}$, em que: QME é o quadrado médio residual;

3. Peso de Akaike (MOTULSKY \& CHRISTOPOULOS, 2003), dado por:

probabilidade $=\frac{\exp (-0,5 \Delta)}{1+\exp (-0,5 \Delta]}$, em que: $\Delta$ a diferença entre os valores do critério de informação de Akaike corrigido (AICc). O peso de Akaike fornece uma probabilidade de que o modelo com menor AICc seja o modelo correto. O valor do AICc foi obtido por meio da expressão:

$A I C c=n \ln \left(\frac{S Q E}{n}\right)+2(q+1)+\frac{2(q+1)(q+2]}{n-(q+2)}$, em que: o último termo da expressão é o termo de correção, sugerido para pequenas amostras e SQE é a soma de quadrado residual.

\section{RESULTADOS E DISCUSSÃO}

$\mathrm{Na}$ tabela 1 estão apresentadas as estimativas dos parâmetros e avaliadores da qualidade de ajuste dos modelos, logístico e gompertz, para as variáveis diâmetro longitudinal externo (DEL) e diâmetro transversal externo (DET) dos frutos de coqueiro da variedade anão verde considerando as estruturas de erros independentes e autoregressivos.

Verifica-se que, para o ajuste dos modelos ao diâmetro longitudinal externo considerando a estrutura de erros independentes (DEL sem AR), o valor da estatística DW foi de aproximadamente 0,64 para o modelo logístico e 0,34 para o modelo gompertz. Ambos os valores são significativos (p-valor <0,0001) para o teste de autocorrelação positiva, indicando a necessidade de considerar um processo autorregressivo (AR), em princípio de primeira ordem, para a série de resíduos.

Ao considerar o vetor de resíduos defasados como uma covariável indexada pelo parâmetro $\rho_{1}$ e proceder à estimação conjunta dos parâmetros verifica-se que as novas estatísticas do teste $\mathrm{DW}$ foram de 1,77 , para o modelo logístico (p-valor $=0,1100$ ), e

Ciência Rural, v.43, n.5, mai, 2013. 
Tabela 1 - Ajuste dos modelos logístico e gompertz aos dados de diâmetro externo longitudinal (DEL) e transversal (DET) de frutos de coqueiro anão verde considerando a estrutura de erros independentes e autoregressivos.

\begin{tabular}{|c|c|c|c|c|}
\hline \multirow{2}{*}{ Estimativas/Avaliadores } & \multicolumn{2}{|c|}{---------------DEL sem AR---------------- } & \multicolumn{2}{|c|}{---------------DEL com AR1-------------- } \\
\hline & Logístico & Gompertz & Logístico & Gompertz \\
\hline Parâmetro A & $21,84(0,35)$ & $25,07(1,09)$ & $21,56(0,26)$ & $23,71(0,49)$ \\
\hline Parâmetro B & $10,82(0,65)$ & $2,96(0,13)$ & $11,11(0,54)$ & $3,10(0,09)$ \\
\hline Parâmetro k & $0,0141(0,0005)$ & $0,0076(0,0006)$ & $0,0144(0,0004)$ & $0,0084(0,0003)$ \\
\hline$\rho_{1}$ & --- & --- & $0,74(0,17)$ & $0,93(0,12)$ \\
\hline$R_{a j}^{2}$ & 0,9960 & 0,9904 & 0,9976 & 0,9973 \\
\hline DPR & 0,4158 & 0,6454 & 0,3156 & 0,3351 \\
\hline DW & 0,6357 & 0,3441 & 1,7793 & 1,9203 \\
\hline $\operatorname{Pr}<\mathrm{DW}$ & $<0,0001$ & $<0,0001$ & 0,1100 & 0,1837 \\
\hline $\mathrm{AICc}$ & $-38,9140$ & $-16,0544$ & $-48,8717$ & $-45,8686$ \\
\hline Peso de Akaike $\left(\Delta=A I C c_{L}-A I C c_{G}\right)$ & 0,9999 & ,8596) & 0,8178 & ,0031) \\
\hline \multirow{2}{*}{ Estimativas/Avaliadores } & \multicolumn{2}{|c|}{---------------DET sem AR---------------- } & \multicolumn{2}{|c|}{-----------DET com AR2------------- } \\
\hline & Logístico & Gompertz & Logístico & Gompertz \\
\hline Parâmetro A & $15,98(0,65)$ & $18,92(1,93)$ & $15,35(0,24)$ & $17,50(0,57)$ \\
\hline Parâmetro B & $11,84(1,92)$ & $2,93(0,27)$ & $13,93(1,07)$ & $3,16(0,13)$ \\
\hline Parâmetro k & $0,0144(0,0013)$ & $0,0073(0,0012)$ & $0,0160(0,0006)$ & $0,0084(0,0005)$ \\
\hline$\rho_{1}$ & --- & --- & $1,13(0,20)$ & $1,34(0,18)$ \\
\hline$\rho_{2}$ & --- & --- & $-0,22(0,12)$ & $-0,44(0,20)$ \\
\hline$R_{a j}^{2}$ & 0,9731 & 0,9561 & 0,9976 & 0,9971 \\
\hline DPR & 0,7909 & 1,0109 & 0,2280 & 0,2489 \\
\hline DW & 0,1733 & 0,1590 & 2,4424 & 2,7573 \\
\hline $\operatorname{Pr}<\mathrm{DW}$ & $<0,0001$ & $<0,0001$ & 0,6263 & 0,8757 \\
\hline $\mathrm{AICc}$ & $-5,4771$ & 7,2794 & $-59,6389$ & $-55,4107$ \\
\hline Peso de Akaike $\left(\Delta=A I C c_{2}-A I C c_{G G}\right)$ & \multicolumn{2}{|c|}{$0,9983(-12,7565)$} & \multicolumn{2}{|c|}{$0,8923(-4,2282)$} \\
\hline
\end{tabular}

$P_{1}$ - parâmetro autorregressivo de primeira ordem; $P_{2}$ parâmetro autoregressivo de segunda ordem; Pr $<\mathrm{DW}$ - valor $\mathrm{p}$ para o teste de autocorrelação positiva; DPR - desvio padrão residual; DW - estatística do teste de Durbin-Watson; AICc - Critério de Informação de

de 1,92 , para o modelo gompertz ( $\mathrm{p}$-valor $=0,1837$ ) e ambos os casos indicam a não necessidade de se considerar um processo autorregressivo de segunda ordem (AR2).

A incorporação do processo AR1 no procedimento de ajuste dos modelos ao DEL (Tabela 1) resultou em alterações muito pequenas nas estimativas dos parâmetros do modelo, sendo que a maior alteração, quando comparado ao modelo sem AR, foi verificada para o parâmetro $\mathrm{K}$ do modelo gompertz que sofreu um acréscimo de $10 \%$ em sua estimativa. Apenas o parâmetro A sofreu redução em sua estimativa (1\% para o modelo logístico e 5\% para o modelo gompertz) ao considerar o AR1 na modelagem.
Para as estimativas dos erros padrões assintóticos dos parâmetros, as reduções forambastante expressivas ao considerar o processo autoregressivo. Para o parâmetro A do modelo gompertz verifica-se uma redução em 55\% na estimativa do erro padrão assintótico. Esse mesmo comportamento foi relatado por MAZZINI et al. (2005), PEREIRA et al. (2005), MENDES et al. (2008) e TERRA et al. (2010). Os autores consideraram, no ajuste de modelos não lineares, a estrutura autoregressiva de primeira e/ ou segunda ordem para o vetor residual e obtiveram estimativas mais precisas dos parâmetros.

Em relação à comparação dos modelos, considerando a estrutura AR1, verifica-se que os 
avaliadores de qualidade de ajuste foram bastante semelhantes, ou seja, ambos os modelos apresentaram altos valores para o coeficiente de determinação ajustado $\left(R_{a j}^{2}\right)$, baixos valores para o desvio padrão residual (DPR) e valores próximo para o critério de informação de Akaike corrigido. Essas pequenas diferenças nos avaliadores de qualidade de ajuste podem causar dúvida ao pesquisador em escolher o modelo que melhor represente o comportamento de seus dados. E é nesse contexto que MOTULSKY \& CHRISTOPOULOS (2003) sugerem a utilização dos pesos de Akaike para proceder a comparação dos modelos em estudo. Os autores citam que se os valores de AICc para dois modelos estão próximos, não existem evidências claras para escolher um modelo em detrimento de outro. Já os pesos de Akaike fornecem uma medida de probabilidade de escolha do modelo correto. Para a variável DEL o valor do peso de Akaike foi de 0,8178, ou seja, o modelo logístico, que apresenta o menor valor de AICc, tem aproximadamente $82 \%$ chance de ser o modelo correto.

Em relação ao ajuste dos modelos logístico e gompertz aos dados de diâmetro transversal externo (DET) dos frutos, considerando a estrutura de erros independentes e autoregressivos, verifica-se que o valor da estatística DW foi de 0,17 para o modelo logístico e 0,16 para o modelo gompertz. Ambos os valores são significativos ( $p$-valor $<0,0001$ ) para o teste de autocorrelação positiva e portanto considerou-se um processo AR. Para essa variável foi necessário o ajuste de um modelo AR2 para que as novas estatísticas do teste DW (2,44 para o modelo logístico e 2,76 para o modelo gompertz) fossem não significativas (valor- $\mathrm{p}=0,6263$ para $\mathrm{o}$ modelo logístico e valor-p=0,8757 para o modelo gompertz).

Em relação à incorporação do AR2 no procedimento de ajuste dos modelos ao DET também foram verificadas pequenas alterações, embora maiores do que as observadas para variável DEL. As maiores alterações foram constatadas no parâmetro B do modelo logístico (aumento de 18\% no valor da estimativa) e no parâmetro $\mathrm{K}$ do modelo gompertz (aumento de 15\% no valor da estimativa). Também para o DET, apenas o parâmetro A sofreu redução em sua estimativa. Para as estimativas dos erros padrões assintóticos dos parâmetros, as reduções foram ainda mais expressivas, quando comparado aos da variável DEL, ao considerar o AR2. Para o parâmetro A do modelo gompertz a redução foi de $70 \%$ no valor da estimativa.

Os avaliadores de qualidade de ajuste dos modelos ao DET dos frutos, da mesma forma que para a variável DEL, foram bastante semelhantes e demonstram um bom ajuste de ambos os modelos. O valor do peso de Akaike, para essa variável, foi de 0,8923 , ou seja, o modelo logístico tem aproximadamente $89 \%$ chance de ser o modelo correto.

$\mathrm{O}$ ajuste do modelo logístico aos dados de diâmetros, longitudinal e transversal, dos frutos de coqueiro anão, considerando a estrutura residual adequada (AR1 para variável DELe AR2 para a variável DET), pode ser visualizado na figura 1 . Verifica-se, por meio da figura que o diâmetro longitudinal externo, ao longo de todo o período avaliado, sempre esteve acima do diâmetro transversal externo indicando que o fruto em todo o período apresentou um formato ovóide. MACIEL et al. (2009) estudaram a caracterização física dos frutos de seis cultivares de coqueiro anão em diferentes estádios de desenvolvimento, dentre elas a cultivar anão verde e relatam o mesmo tipo de comportamento para essas variáveis.

Em relação à estabilização dos diâmetros verifica-se que o DEL começa a se estabilizar em torno de 300 dias (10 meses) após a abertura da inflorescência, quando o fruto apresenta um valor estimado de $21,56 \mathrm{~cm}$. Já o DET começa a se estabilizar em torno dos 270 dias ( 9 meses) e nessa época o fruto apresenta um valor estimado de $15,35 \mathrm{~cm}$.

CORRÊA et al. (2002) avaliando frutos de coqueiro anão verde, cultivados sob irrigação, obtiveram valores médios de $16,64 \mathrm{~cm}$ e $13,07 \mathrm{~cm}$ para o comprimento e largura dos frutos, respectivamente. ARAGÃO et al. (2002) encontraram valores médios de $22,69 \mathrm{~cm}$ de comprimento e $17,20 \mathrm{~cm}$ de largura, em frutos de coqueiros híbridos. SILVA et al. (2009) estudando características biométricas de frutos de coqueiro anão verde, com sete meses deidade (210 dias), obteve valores de $17,23 \mathrm{~cm}$ e $14,97 \mathrm{~cm}$ para o diâmetro longitudinal e transversal do fruto, respectivamente, no sistema de produção convencional.

Em geral, os pesquisadores que estudam as características biométricas de frutos, conduzem os experimentos de avaliação até por volta de 210240 dias após a abertura de inflorescência e o que se verifica é que os frutos mantidos na planta continuam a completar seu crescimento até períodos mais tardios. Portanto, deve-se ter cautela ao sugerir o ponto ideal de colheita dos frutos apenas com base nas medidas do fruto à maturação. RESENDE et al. (2002) relatam que o coco verde apresenta ponto ideal de colheita quando a água já desenvolveu todas as características sensoriais que a tornem apta para o consumo. BENASSI (2007) por meio da avaliação sensorial da água de coco em frutos da variedade anão verde encontrou propriedades desejáveis do produto para frutos com idade entre 8 e 11 meses. FONTES et 


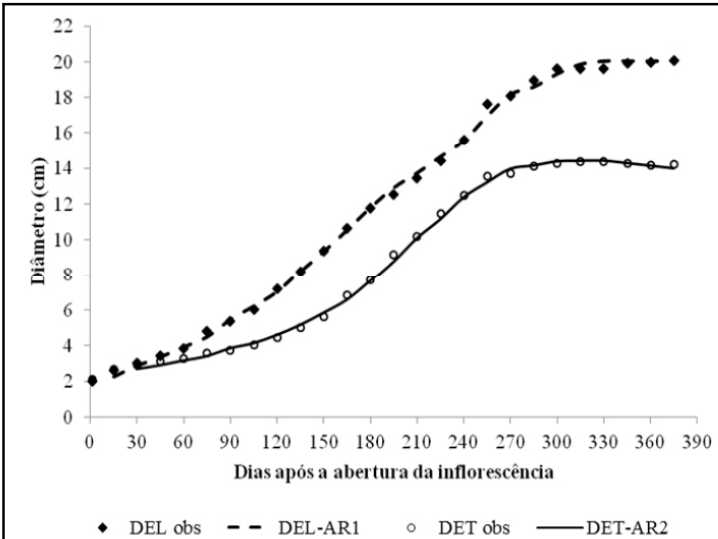

Figura 1 - Valores observados e estimados, por meio do modelo logístico, do diâmetro longitudinal externo (DEL) e diâmetro transversal externo (DET) de frutos de coqueiro da variedade anão verde.

al. (1998) e RESENDE et al. (2002) concordam que quando a produção se destina ao consumo in natura da água-de-coco, este deve ser colhido entre os seis e oito meses de idade.

\section{CONCLUSÃO}

A consideração da estrutura residual adequada no ajuste dos modelos logístico e gompertz resultaram em reduções expressivas nas estimativas dos erros padrões assintóticos dos parâmetros, sendo o modelo logístico, o mais adequado para descrever o crescimento, em diâmetro, longitudinal e transversal, de frutos de coqueiro anão verde. De acordo com o ajuste, o fruto, totalmente desenvolvido, tem diâmetros externos, longitudinal e transversal, de $21,56 \mathrm{~cm}$ e $15,35 \mathrm{~cm}$, respectivamente.

\section{REFERÊNCIAS}

ARAGÃO, W.M. Cultivares de coqueiros. In: FONTES, H.R. et al. (Ed.). A cultura do coqueiro. Aracaju: Embrapa Tabuleiros Costeiros, 2007. (Embrapa Tabuleiros Costeiros. Sistemas de Produção, 1). Disponível em: <http://sistemasdeproducao.cnptia. embrapa.br/FontesHTML/Coco/ACulturadoCoqueiro/cultivares. htm>. Acesso em: 6 ago. 2011.

ARAGÃO, W.M. et al. Fruto do coqueiro para consumo natural. In: ARAGÃO, W.M. (Ed.). Coco pós-colheita. Brasília: EMBRAPA, 2002. p.19-25. (Série Frutas do Brasil, 29).

BENASSI, A.C. Caracterizações biométricas, química e sensorial de frutos de coqueiro variedade anã verde. 2006. 49f. Tese (Doutorado em Produção Vegetal) - Universidade Estadual Paulista "Júlio de Mesquita Filho", Jaboticabal.

BENASSI, A.C. et al. Caracterização biométrica de frutos de coqueiro, Cocos nucifera L. variedade anã-verde, em diferentes estádios de desenvolvimento. Revista Brasileira Fruticultura, v.29, n.2, p.302-307, 2007. Disponível em: $<$ http://www.scielo.br/scielo.php?script=sci_arttext\&pid=S0100$29452007000200022 \& \operatorname{lng}=e n \& n r m=i s o>$. Acesso em: 23 ago. 2011. doi: 10.1590/S0100-29452007000200022.

BITENCOURT, D.V.; PEDROTI, A. Usos da casca de coco: estudo das viabilidades de implantação de usina de beneficiamento de fibra de coco em Sergipe. Revista da FAPESE de Pesquisa e Extensão, v.4, p.115-124, 2008

CORRÊA, L.S. et al. Comportamento do coqueiro anão verde (Cocos nucifera L.) em Selvíria, MS. In: CONGRESSO BRASILEIRO DE FRUTICULTURA, 17., 2002, Belém, PA. Anais... Belém: Sociedade Brasileira de Fruticultura, 2002. CD-ROM.

FONTES, H.R. et al. Implantação e manejo da cultura do coqueiro. In: FERREIRA, J.M.S. et al. (Ed.). A cultura do coqueiro no Brasil. 2.ed. Aracaju: Embrapa-SPI, 1998. p.99-128.

MACIEL, V.T. et al. Caracterização física dos frutos de seis cultivares de coqueiro anão verde em diferentes estádios de desenvolvimento. Revista Brasileira de Ciências Agrárias, v.4, n.4, p.395-398, 2009.

MAZZINI, A.R. de A. et al. Curva de crescimento de novilhos Hereford: heterocedasticidade e resíduos autoregressivos. Ciência Rural, v.35, p.422-427, 2005. Disponível em: <http://www.scielo. $\mathrm{br} / \mathrm{scielo}$.php? pid=S0103-84782005000200028\& $\mathrm{script}=\mathrm{sci}_{-}$ arttext>. Acesso em: 21 fev. 2011. doi: 10.1590/S010384782005000200028 .

MEDINA, J.C. Coco I - Cultura. In: MEDINA, J.C. et al. (Ed.). Coco da cultura ao processamento e comercialização. São Paulo: ITAL, 1980. p.7-172.

MENDES, P.N. et al. Modelo logístico difásico no estudo do crescimento de fêmeas da raça Hereford. Ciência Rural, v.38, n.7, p. 1984-1990, 2008. Disponível em: <http://www.scielo.br/scielo. php?script=sci_arttext\&pid=S0103-84782008000700029\&lng =pt\&nrm=iso $>$. Acesso em: 23 ago. 2011. doi: 10.1590/S010384782008000700028 .

MOKKADEM, A. Le modele non linéaire AR(1) general. Ergodicité et ergodicité géométrique. Comptes Rendues Academic Scientifique Paris, v.301, Série I, p.889-892, 1985.

MORETTIN, P.A.; TOLOI, C.M. de C. Previsão de séries temporais. 3.ed. São Paulo: Atual, 2004. 436p.

MOTULSKY, H.; CHRISTOPOULOS, A. Fitting models to biological data using linear and nonlinear regression: a practical guide to curve fitting. 4.ed. San Diego, CA: GraphPad Software, 2003. $351 \mathrm{p}$.

PEREIRA, J.M. et al. Nonlinear models to predict nitrogen mineralization in an Oxisol. Scientia Agricola, v.62, n.4, p.548-554, 2005. Disponível em: <http://www.scielo.br/scielo. php?pid=S0103-0162005000400014\&script=sci_arttext $>$. Acesso em: 21 fev. 2011. doi: 10.1590/S0103-90162005000400014.

RESENDE, J.M. et al. Colheita e manuseio pós-colheita. In: ARAGÃO, W.M. (Ed.). Coco pós colheita. Brasília: EMBRAPA, 2002. p.35-41. (Série Frutas do Brasil, 29).

SAS Institute. SAS Procedures guide for computers. 6.ed. Cary, NC, 1999. V.3, 373p. 
SAVIAN, T.V.; MUNIZ, J.A. A study of in situ degradability: heterogeneity of variances and correlated errors. Scientia Agricola, v.64, p.548-554, 2007. Disponível em: <http://www.scielo.br/ scielo.php?script=sci_arttext $\&$ pid=S0103-90162007000500013>. Acessoem: 22 fev. 2011. doi: 10.1590/S0103-90162007000500013.

SILVA, D.L.V. da et al. Características físicas, físico-químicas e sensoriais da água de frutos de coqueiro anão verde oriundo de produção convencional e orgânica. Ciência e Agrotecnologia, v.33, n.4, p.1079-1084, 2009. Disponível em: <http:// www.scielo.br/scielo.php?script $=$ sci_arttext \&pid $=$ S 1413 $70542009000400019 \& \operatorname{lng}=\mathrm{en} \& \mathrm{nrm}=\mathrm{iso}>$. Acesso em: 24 maio, 2012. doi: 10.1590/S1413-70542009000400019.

SOUZA, G. da S. Introdução aos modelos de regressão linear e não-linear. Brasília: Embrapa- SPI/Embrapa-SEA, 1998. 489p.

TERRA, M.F. et al. Ajuste dos modelos Logístico e Gompertz aos dados de crescimento de frutos da tamareira-anã (Phoenix roebelenii O'BRIEN). Magistra, v.22, n.1, p.01-07, 2010. 